\title{
An automated system for administering continuous workload and for measuring sustained continuous performance
}

\author{
DANIEL J. MULLANEY, PAUL A. FLECK, NOBUYUKI OKUDAIRA, and DANIEL F. KRIPKE \\ University of California, San Diego, La Jolla, California \\ and San Diego Veterans Administration Medical Center, San Diego, California
}

\begin{abstract}
Sustained continuous performance for up to $42 \mathrm{~h}$ was studied with 60 male volunteers in two separate protocols. The recuperative value of six 1-h nap breaks and a single 6-h nap break were contrasted in 20 subjects, 10 in each nap group. Forty other subjects attempted to work continuously with no breaks for $42 \mathrm{~h}$. Twenty of these subjects worked simultaneously on separate parallel computer-based tasks, but worked in the same room in pairs. All subjects in the two nap groups ( $\mathrm{N}=10$ and $\mathrm{N}=10$ ), as well as 20 who had no scheduled breaks, worked alone, almost isolated, with minimal interaction with the experimenters. During each $10 \mathrm{~min}$, subjects performed a tracking task, a pattern-memory task, and an addition task and provided subjective ratings on sleepiness and attention/fantasy. Results showed that computerized tasks demanding sustained continuous performance without naps cause more rapid performance deterioration than previously tested intermittent-work paradigms.
\end{abstract}

The availability of microprocessors and computerized video games has made possible a new approach to the conduct of psychophysiological experiments, especially in some models of crucial work situations. Few previous studies have examined the psychological effects of truly continuous sustained performance for $12 \mathrm{~h}$ or more. In this report, we examine continuous sustained performance whereby subjects are tested for $9+$ of every $10 \mathrm{~min}, 24$ h a day (Kripke, Fleck, Mullaney, \& Levy, 1983; Mullaney, Kripke, Fleck, \& Okudaira, 1983; Mullaney, Kripke, Fleck, \& Johnson, 1983).

\section{COMPUTER SYSTEM}

A commercial home computer system (Apple II) was adapted to continuously present performance tasks. One task was an adaptation of a joystick-controlled tracking game, another was a video pattern-memory task, and one involved keyboard addition.

In the first protocol, task duration for each of the three performance tests was $3.0 \mathrm{~min}$. The first task, tracking, displayed a small randomly moving target on a video monitor screen that also displayed a stationary grid. The simulation resembled a pilot guiding a moving target into his

This research was supported by Office of Naval Research Contract N00014-79-C-0317, Department of Army Medical Defense Contract 17-78-C-8040, National Institute of Mental Health Research Scientist Development Award K02 MH0017 to Daniel F. Kripke, and the Research Service of the Veterans Administration. We thank Laverne C. Johnson, William Mason, John Webster, Martha Martell, and Sam Messin for their assistance. Address correspondence and requests for reprints to D. Mullaney, Department of Psychiatry (116A), San Diego Veterans Administration Medical Center, 3350 La Jolla Village Drive, San Diego, CA 92161. gunsights. Using a control stick, the subject was required to position the target in the center of the stationary grid, at which time he could score a "hit" on the target by pressing a "trigger" button. A simulated explosion provided immediate feedback when the target was properly tracked and hit. A new target then appeared on the screen in a random position. The number of "hits" was tabulated by the computer. This task was self-paced, and instructions to subjects were to score as many "hits" as possible.

In the second task, pattern memory, a randomly generated $5 \times 5$ matrix of 25 rectangles (either white or black, measuring $2.6 \times 3.7 \mathrm{~mm}$ ) was displayed on the video monitor screen for $3 \mathrm{sec}$. After this initial pattern had disappeared from the screen, a second and a third pattern were sequentially displayed, either of which might be identical to the original pattern or might differ randomly in one of the 25 rectangles. Subjects indicated by pressing the appropriate key whether the second, the third, or neither copy was identical to the first pattern. This task was experiment-paced, and $3.2 \mathrm{sec}$ were allowed for responses. Subjects received immediate feedback on whether their identifications were correct or incorrect.

In the third task, addition, subjects were required to add two randomly generated three-digit numbers (presented on the monitor), add 7, and then enter the threedigit sum on a numeric keyboard. The time allowed for responses was limited, and the limit was varied adaptively in proportion to an individual's performance success. This task was experiment-paced, although adaptive to an individual's ability, since time allowed for responses varied with performance level, and this tended to demand maximal performance speed. Subjects were given immediate feedback regarding "correct" and "wrong" additions. Speed and accuracy were both emphasized. Earned 
bonuses depended on the number of correct additions. The computer system was programmed to make repeated queries of the subjects, immediately following the addition task, to record behaviors that might influence performance, such as eating, drinking, and restroom visits. Subjective states along two visual-analog scales measuring continua from sleepiness to alertness and from perceptual attentiveness to daydreaming were also recorded. Thus, since performance tests occupied only 9 of every $10 \mathrm{~min}$, subjects were kept busy during the 10th min with subjective data entries.

At the end of each task, the video monitor displayed summary feedback on performance in the form of a statement of the amount of bonus money earned during the 3-min task interval. The performance tests were framed as a repetitive series of tasks in which correct performance was rewarded with money. Failures to respond to the task presentations were scored by the computer in a way that heavily penalized inattention or sleep but less heavily penalized errors representing a continuing effort. During a 10-min period, a subject would actually lose previously earned bonus money for no responses but would earn some by making one correct response to each task. The program also generated alerting signals for failure to respond to task demands. In this way, the reward system elicited highly motivated effort from most subjects.

In the second protocol, performance task durations were shortened to $2.5 \mathrm{~min}$ for each of the three sequential tasks to allow more time at the end of each 10-min segment for subjects to record subjective data. The program interrogated subjects as in the first protocol, recorded responses, and then displayed summary performance feedback plus a random three-digit code number with instructions to record this number on a $5 \times 7$ in. card together with a brief description of "what has been on your mind over the past 10 minutes." The subject then contributed a free verbal report.

During each $10 \mathrm{~min}$, subjects performed the same three tasks and provided subjective data. The operating system of the microcomputer was adapted in conjunction with a real-time clock to allow real-time control of the sequence of task presentation, the presentation of tasks, and automatic scoring of performance results, all at the same time. Most of the program was written in BASIC. Interrupts and real-time interactions with subjects were written in 6502 assembly.

\section{PROCEDURE}

The microcomputer-based system was used to study sustained continuous performance with 60 male volunteers in protocols lasting $48 \mathrm{~h}$. All studies began with a 6-h sleep period, which was chosen to be as close as possible to the subjects' usual bedtime. This produced a standardized prior sleep state. Right- and left-hemisphere parietooccipital EEG, submental electromyogram (EMG), bipolar horizontal electrooculogram (EOG), and wrist activity from the dominant wrist were recorded during sleep.
All subjects in the first protocol (which included both groups that were allowed nap breaks) wore recording electrodes during the entire study in order to examine sleepstage patterning during naps and to measure unscheduled microsleeps. Forty subjects attempted to work continuously for $42 \mathrm{~h}$ with no breaks. Of these, 20 paired subjects worked continuously on similar computer consoles in the same room. Twenty of the other subjects worked in semi-isolation, with minimal contact with the experimenters. The recuperative value of six $1-\mathrm{h}$ nap breaks was examined in 10 subjects, and that of a single 6-h nap break was examined in an additional 10 subjects. Subjects in both nap groups were isolated and worked alone. The subjects in the 1-h nap group were given opportunities to rest after every $6 \mathrm{~h}$ of performance and were encouraged to sleep during this hour. The 6-h nap group performed for $18 \mathrm{~h}$, were given a 6-h rest and encouraged to sleep, and then were asked to perform for another $18 \mathrm{~h}$.

\section{DATA SUMMARY}

Our studies (Kripke, et al., 1983; Mullaney, Kripke, Fleck \& Johnson, 1983; Mullaney, Kripke, Fleck \& Okudaira, 1983) showed that psychological disturbances developed more rapidly during sustained performance in the computerized experiment than they had in previous experiments that had focused primarily on paper-andpencil tasks, generally with substantial breaks between testing sessions (Johnson \& Naitoh, 1974; Kollar, Slater, Palmer, Doctor, \& Mandell, 1966; Opstad, Ekanger, Nummestrad, \& Rabbe, 1978).

Of the 40 motivated subjects asked to work continuously for $42 \mathrm{~h}$ with no breaks, 12 were unable to complete the computer-driven protocol. Working in pairs did not significantly lessen the dropout rate. More than half of our subjects experienced notable psychological disturbances, such as mild visual hallucinations, illusions, and derealizations. Subjects working for 42 hours with no breaks experienced significantly more of these disturbances than those who were allowed naps. The incidence of psychological disturbances in paired subjects working for $42 \mathrm{~h}$ continuously was not significantly different from the incidence for subjects working alone. Anecdotally, subjects in pairs reported that social interactions helped reorient them, lessened disturbances, and allowed steadier performance, but our objective data do not corroborate these subjective impressions. Few disturbances occurred in the first $14 \mathrm{~h}$ of work, and $80 \%$ of the psychological disturbances occurred after $18 \mathrm{~h}$ of performance (Mullaney, Kripke, Fleck, \& Okudaira, 1983). Surprisingly, we found no obvious, measurable relationship between psychological disturbances, such as hallucinations, and performance decrements on a minute-by-minute basis.

Continuous sustained performance produced rapid fatigue effects detectable after only $6 \mathrm{~h}$. Performance showed large circadian-rhythm (24-h) effects in all groups; the poorest performance occurred during the subjects' normal time for sleep (approximately $2300-0700 \mathrm{~h}$, on aver- 
age). A circadian effect was observed even in the group allowed $1-h$ rests for sleep every $7 h$. One-hour naps were of benefit in sustaining performance throughout the 42-h period (especially during the initial $18 \mathrm{~h}$ ), but subjects allowed one 6-h break for sleep after $18 \mathrm{~h}$ of testing performed better than all others during the last $18 \mathrm{~h}$ of the 42-h testing period.

Sustained continuous performance without sleep appeared to be the major factor producing performance deterioration and psychological disturbances. Minimal social contact did not significantly affect performance or the onset of disturbances. More enhanced social contacts requiring joint effort or communication between subjects for task performance should be investigated to determine whether these factors reduce psychological disturbances and performance deterioration.

\section{MICROPROCESSORS IN RESEARCH}

Computer technology creates new challenges to human performance. Computers can work continuously without breaks and may help create situations in which people are driven to work continuously as well. Automated testing can also be used to more clearly examine the problems that develop during continuous sustained tasks. As computer systems have become more commonplace in or- dinary life, new tools for automatic testing and data collection have proliferated. Increasingly, with only slight software modification, commercially available computer systems can be used to model modern job situations and simultaneously to record a variety of physiological and behavioral data.

\section{REFERENCES}

JoHnson, L. C., \& NAITOH, P. (1974). The operational consequences of sleep deprivation and sleep deficit (NATO/AGARDograph AGARD-AG-193). London: Harford House.

Kollar, E. J., Slater, G. R., Palmer, J. O., Doctor, R. F., \& MaNDELL, A. J. (1966). Stress in subjects undergoing sleep deprivation. Psychosomatic Medicine, 28, 101-113.

Kripke, D. F., Fleck, P. A., Mullaney, D. J., \&evy, M. L. (1983). Behavioral analogs of the REM-nonREM cycle. In J. Mendlewicz \& H. M. Praag (Eds.), Advances in biological psychiatry (pp. 7279). Basel, Switzerland: Karger.

Mullaney, D. J., Kripke, D. F., Fleck, P. A., Johnson, L. C. (1983). Sleep loss and nap effects on sustained continuous performance. Psychophysiology, 20, 643-651.

Mullaney, D. J., Kripke, D. F., Fleck, P. A., \& Okudaira, N. (1983). Effects of sustained continuous performance on subjects working alone and in pairs. Perceptual and Motor Skills, 57, 819-832.

Opstad, P. K., Ekanger, M., Nummestrad, M., \& RabBe, N. (1978). Performance mood, and clinical symptoms in men exposed to prolonged, severe work and sleep deprivation. Aviation, Space and Environmental Medicine, 49, 1065-1073. 